\title{
A Class of Nonautonomous Schistosomiasis Transmission Model with Incubation Period
}

\author{
Yang Liu1, Yuying He², Shuixian Yan ${ }^{1 *}$, Shujing Gao \\ ${ }^{1}$ College of Mathematics and Computer Science, Gannan Normal University, Ganzhou, China \\ ${ }^{2}$ Yuhua Elite School, Kaifeng, China \\ Email: *yanshuixian1017@163.com
}

How to cite this paper: Liu, Y., He, Y.Y., Yan, S.X. and Gao, S.J. (2019) A Class of Nonautonomous Schistosomiasis Transmission Model with Incubation Period. Applied Mathematics, 10, 159-172. https://doi.org/10.4236/am.2019.103013

Received: December 21, 2018

Accepted: March 26, 2019

Published: March 29, 2019

Copyright (c) 2019 by author(s) and Scientific Research Publishing Inc. This work is licensed under the Creative Commons Attribution International License (CC BY 4.0).

http://creativecommons.org/licenses/by/4.0/

\begin{abstract}
A nonautonomous schistosomiasis model with latent period and saturated incidence is investigated. Further, we study the long-time behavior of the epidemic model. The weaker sufficient conditions for the permanence and extinction of infectious population of the model are obtained by constructing some auxiliary functions. Numerical simulations show agreement with the theoretical results.
\end{abstract}

\section{Keywords}

Schistosomiasis, Nonautonomous Model, Permanence, Extinction

\section{Introduction}

Schistosomiasis (also known as bilharzia) is a disease caused by parasitic worms of the Schistosoma type [1]. Schistosomiasis affects almost 210 million people worldwide [2], and an estimated 12,000 to 200,000 people die from it each year [3] [4]. The disease is most commonly found in Africa, Asia and South America [5]. Around 700 million people, in more than 70 countries, live in areas where the disease is common [4] [6]. Schistosomiasis is second only to malaria, as a parasitic disease with the greatest economic impact [7].

Mathematical modeling has become an important tool in analyzing the spread and control of infectious diseases. In recent years, many schostosomiasis models have been proposed and studied ([8]-[13], etc.). These models provide a detailed exposition on how to describe, analyze, and predict epidemics of schistosomiasis for the ultimate purposes of developing control strategies and tactics for schistosomiasis transmission.

Many diseases incubate inside the hosts for a period of time before the hosts 
become infectious. Using a compartmental approach, one may assume that a susceptible individual first goes through an incubation period (and is said to become exposed or in the class $E$ ) after infection, before becoming infectious. The resulting models are of SEIR or SEIRS types, respectively, depending on whether the acquired immunity is permanent or otherwise.

In the aforementioned framework, their coefficients are considered as constants, which are approximated by average values. However, we note that ecosystems in the real world often appear the nonautonomous phenomenon. Recently many nonautonomous epidemic systems have been studied ([14]-[20], etc.). In fact, natural factors, such as seasonal changes in moisture and temperature, affect the abundance and activity of the intermediate snail host, Oncomelania hupensis, and the transmission dynamics of schistosomiasis are in a constant state of flux [21]. Moreover, there are many social factors related to human behaviors accounting for the change of schistosomiasis incidence, such as marked changes of contact rates caused by daily production activities [22]. This illustrates that the transmission of schistosomiasis shows seasonal behavior. In order to describe this kind of phenomenon, in the model, the parameters of the system should be functions of time. As far as we know, the research work on the nonautonomous schistosomiasis models is very few. Therefore, it is necessary to study nonautonomous schistosomiasis models.

In this paper, we assume large intermediate host population and thus ignore snail dynamics. Motivated by the above description, we develop a class of nonautonomous schistosomiasis transmission model with incubation period:

$$
\left\{\begin{array}{l}
\frac{\mathrm{d} S(t)}{\mathrm{d} t}=\Lambda(t)-\frac{\beta(t) S(t) I(t)}{1+\alpha S(t)}-\mu(t) S(t)+\delta(t) I(t)+\gamma(t) E(t), \\
\frac{\mathrm{d} E(t)}{\mathrm{d} t}=\frac{\beta(t) S(t) I(t)}{1+\alpha S(t)}-(\mu(t)+\varepsilon(t)+\gamma(t)) E(t), \\
\frac{\mathrm{d} I(t)}{\mathrm{d} t}=\varepsilon(t) E(t)-(\mu(t)+\delta(t)+d(t)) I(t) .
\end{array}\right.
$$

with initial value

$$
S(0)>0, E(0)>0, I(0)>0 .
$$

Here $S(t), E(t)$ and $I(t)$ denote the size of susceptible, exposed, infectious population at time $t$, respectively. $\Lambda(t)$ is the growth rate of population, $\mu(t)$ is the natural death rate of the population, $\beta(t)$ is the rate of the efficient contact, $\delta(t)$ and $\gamma(t)$ are the recovery rates of infectious population and exposed population, respectively, $d(t)$ is the disease-related death rate and $\varepsilon(t)$ is the rate of developing infectivity at time $t$.

The organization of this paper is as follows. In the next section, we present preliminaries setting and propositions, which we use to analyze the long-time behavior of system (1.1) in the following sections. In Section 3, we establish the extinction of the disease of system (1.1). In Section 4, we will discuss the permanence of the infectious population. Our results are verified by numerical simula- 
tions in Section 5

\section{Preliminaries}

In this section, system (1.1) satisfies the following assumptions:

$\left(\mathrm{H}_{1}\right)$ : The functions $\Lambda(t), \delta(t), \mu(t), \delta(t), \gamma(t), \varepsilon(t), d(t)$ are nonnegative, bounded and continuous on $[0,+\infty)$ and $\beta(0)>0$.

$\left(\mathrm{H}_{2}\right)$ : There exist positive constants $\omega_{i}>0(i=1,2,3)$ such that

$$
\begin{aligned}
& \liminf _{t \rightarrow+\infty} \int_{t}^{t+\omega_{2}} \beta(s) \mathrm{d} s>0, \\
& \liminf _{t \rightarrow+\infty} \int_{t}^{t+\omega_{2}} \mu(s) \mathrm{d} s>0, \\
& \liminf _{t \rightarrow+\infty} \int_{t}^{t+\omega_{2}} \Lambda(s) \mathrm{d} s>0 .
\end{aligned}
$$

Adding all the equations of model (1.1), then we have

$$
\begin{aligned}
\Lambda(t)-\mu(t) N(t) & \geq \frac{\mathrm{d} N(t)}{\mathrm{d} t}=\Lambda(t)-\mu(t) N(t)-d(t) I(t) \\
& \geq \Lambda(t)-(\mu(t)+d(t)) N(t)
\end{aligned}
$$

Let $N(t)=S(t)+E(t)+I(t)$ be the total population in system (1.1) with the initial value $N(0)=S(0)+E(0)+I(0)$. We denote by $N^{*}(t)$ the solution of

$$
\frac{\mathrm{d} N^{*}(t)}{\mathrm{d} t}=\Lambda(t)-\mu(t) N^{*}(t)
$$

with initial value (1.2), and denote by $N_{*}(t)$ the solution of

$$
\frac{\mathrm{d} N_{*}(t)}{\mathrm{d} t}=\Lambda(t)-(\mu(t)+d(t)) N_{*}(t)
$$

with initial value (1.2). Then

$$
N_{*}(t) \leq S(t)+E(t)+I(t) \leq N^{*}(t) .
$$

By [22], we have the following result:

Lemma 2.1. Suppose that assumptions $\left(\mathrm{H}_{1}\right)$ and $\left(\mathrm{H}_{2}\right)$ hold. Then:

(i) there exist positive constants $m>0$ and $M>0$, such that

$$
\begin{aligned}
0 & <m \leq \liminf _{t \rightarrow+\infty} N_{*}(t) \leq \limsup _{t \rightarrow+\infty} N_{*}(t) \\
& \leq \liminf _{t \rightarrow+\infty} N^{*}(t) \leq \limsup _{t \rightarrow+\infty} N^{*}(t) \leq M<+\infty .
\end{aligned}
$$

(ii) the solution $(S(t), E(t), I(t))$ of system (1.1) with the initial value (1.2) exists, is uniformly bounded and $S(t)>0, E(t)>0, I(t)>0$ for all $t>0$. For the solution $(S(t), E(t), I(t))$ of system (1.1), we define

$$
G(p, t):=\frac{p \beta(t) S(t)}{1+\alpha S(t)}+\delta(t)+d(t)-\gamma(t)-\left(1+\frac{1}{p}\right) \varepsilon(t)
$$

and

$$
W(p, t):=p E(t)-I(t) .
$$

for $p>0, t>0$. In Sections 3 and 4 we use the following lemma in order to 
investigate the longtime behavior of system (1.1).

Lemma 2.2. If there exist positive constants $p>0$ and $T_{1}>0$ such that $G(p, t)<0$ for all $t \geq T_{1}$, then there exists $T_{2} \geq T_{1}$ such that $W(p, t)>0$ or $W(p, t) \leq 0$ for all $t \geq T_{2}$.

Proof: Suppose that there does not exist $T_{2} \geq T_{1}$ such that $W(p, t)>0$ or $W(p, t) \leq 0$ for all $t \geq T_{2}$. So we have

$$
p E(s)=I(s)
$$

and

$$
\begin{aligned}
\frac{\mathrm{d} W(p, s)}{\mathrm{d} t}= & p\left\{\frac{\beta(s) S(s) I(s)}{1+\alpha S(s)}-(\mu(s)+\varepsilon(s)+\gamma(s)) E(s)\right\} \\
& -\{\varepsilon(s) E(s)-(\mu(s)+\delta(s)+d(s)) I(s)\} \\
= & I(s)\left\{\frac{p \beta(s) S(s)}{1+\alpha S(s)}+(\mu(s)+\delta(s)+d(s))\right\} \\
& -p E(s)\left\{(\mu(s)+\varepsilon(s)+\gamma(s))+\frac{\varepsilon(s)}{p}\right\} \\
> & 0 .
\end{aligned}
$$

Substituting (2.5) into (2.6), we have

$$
\begin{aligned}
0 & <p E(s)\left\{\frac{p \beta(s) S(s)}{1+\alpha S(s)}+\delta(s)+d(s)-\gamma(s)-\left(1+\frac{1}{p}\right) \varepsilon(s)\right\} \\
& =p E(s) G(p, s) .
\end{aligned}
$$

From Lemma 2.1, we have $p>0$ and $E(s)>0$, so $G(p, s)>0$, which is a contradiction with $G(p, t)<0$ for all $t \geq T_{1}$. The proof is completed.

\section{Extinction of Infectious Population}

In this section, we obtain conditions for focus on the extinction of the infectious population of system (1.1).

Theorem 3.1 Suppose that assumptions $\left(\mathrm{H}_{1}\right)$ and $\left(\mathrm{H}_{2}\right)$ hold. If there exist $\lambda>0, p>0$ and $T_{1}>0$ such that

$$
\begin{gathered}
R_{1}(\lambda, p):=\limsup _{t \rightarrow+\infty} \int_{t}^{t+\lambda}\left\{p \frac{\beta(s)}{1+\alpha N^{*}(s)} N^{*}(s)-(\mu(s)+\varepsilon(s)+\gamma(s))\right\} \mathrm{d} s<0, \\
R_{1}^{*}(\lambda, p):=\limsup _{t \rightarrow+\infty} \int_{t}^{t+\lambda}\left\{\varepsilon(s) \frac{1}{p}-(\mu(s)+\delta(s)+d(s))\right\} \mathrm{d} s<0
\end{gathered}
$$

and $G(p, t)<0$ for all $t \geq T_{1}$, then infectious population $I(t)$ in system $(1.1)$ is extinct. i.e.

$$
\lim _{t \rightarrow+\infty} I(t)=0
$$

Proof: From Lemma 2.2, we consider the following two cases:

(i) $p E(t)>I(t)$ for all $t \geq T_{2}$;

(ii) $p E(t) \leq I(t)$ for all $t \geq T_{2}$. 
First, we consider the cases (i). From the second equation of system (1.1), we have

$$
\begin{aligned}
\frac{\mathrm{d} E(t)}{\mathrm{d} t}= & \frac{\beta(t) I(t)}{1+\alpha(N(t)-E(t)-I(t))}(N(t)-E(t)-I(t)) \\
& -(\mu(t)+\varepsilon(t)+\gamma(t)) E(t) \\
< & \frac{p \beta(t) E(t)}{1+\alpha\left(N^{*}(t)-E(t)-I(t)\right)}\left(N^{*}(t)-E(t)-I(t)\right) \\
& -(\mu(t)+\varepsilon(t)+\gamma(t)) E(t) \\
< & \frac{p \beta(t) E(t)}{1+\alpha N^{*}(t)} N^{*}(t)-(\mu(t)+\varepsilon(t)+\gamma(t)) E(t) \\
= & E(t)\left\{\frac{p \beta(t)}{1+\alpha N^{*}(t)} N^{*}(t)-(\mu(t)+\varepsilon(t)+\gamma(t))\right\} .
\end{aligned}
$$

So we have

$$
E(t)<E\left(T_{2}\right) \exp \left(\int_{T_{2}}^{t}\left\{p \frac{\beta(s)}{1+\alpha N^{*}(s)} N^{*}(s)-(\mu(s)+\varepsilon(s)+\gamma(s))\right\} \mathrm{d} s\right)
$$

for all $t \geq T_{2}$. From (3.1), we see that there exist constants $\delta_{1}>0$ and $T_{3}>T_{2}$ such that

$$
\int_{t}^{t+\lambda}\left\{p \frac{\beta(s)}{1+\alpha N^{*}(s)} N^{*}(s)-(\mu(s)+\varepsilon(s)+\gamma(s))\right\} \mathrm{d} s<-\delta_{1}
$$

for all $t>T_{3}$. From (3.3) and (3.4), we obtain $\lim _{t \rightarrow+\infty} E(t)=0$. Therefore, it follows from $p E(t)>I(t)$, that $\lim _{t \rightarrow+\infty} I(t)=0$. Now we consider the case (ii). From $E(t) \leq \frac{I(t)}{p}$ for all $t \geq T_{2}$ and the third equation of (1.1), we have

$$
\frac{\mathrm{d} I(t)}{\mathrm{d} t} \leq I(t)\left\{\varepsilon(t) \frac{1}{p}-(\mu(t)+\delta(t)+d(t))\right\} .
$$

Then the following expression

$$
I(t) \leq I\left(T_{2}\right) \exp \left(\int_{T_{2}}^{t}\left\{\frac{\varepsilon(s)}{p}-\mu(s)-\delta(s)-d(s)\right\} \mathrm{d} s\right)
$$

for all $t>T_{2}$ hold. Hence, by (3.2), there exist $\delta_{2}>0$ and $T_{4}>T_{2}$ such that

$$
\int_{t+\lambda}^{t}\left\{\varepsilon(s) \frac{1}{p}-(\mu(s)+\delta(s)+d(s))\right\} \mathrm{d} s<-\delta_{2},
$$

for $t \geq T_{4}$. From (3.5) and (3.6), we have

$$
\lim _{t \rightarrow+\infty} I(t)=0 \text {. }
$$

\section{Permanence of Infectious Population}

In this section, we obtain the sufficient conditions for the permanence of infectious population. 
Theorem 4.1. Suppose that assumptions $\left(\mathrm{H}_{1}\right)$ and $\left(\mathrm{H}_{2}\right)$ hold. If there are $\lambda>0, p>0$ and $T_{1}>0$ such that

$$
\begin{gathered}
R_{2}(\lambda, p):=\liminf _{t \rightarrow+\infty} \int_{t}^{t+\lambda}\left\{p \frac{\beta(s)}{1+\alpha N_{*}(s)} N_{*}(s)-(\mu(s)+\varepsilon(s)+\gamma(s))\right\} \mathrm{d} s>0, \\
R_{2}^{*}(\lambda, p):=\liminf _{t \rightarrow+\infty} \int_{t}^{t+\lambda}\left\{\varepsilon(s) \frac{1}{p}-(\mu(s)+\delta(s)+d(s))\right\} \mathrm{d} s>0
\end{gathered}
$$

and $G(p, t)<0$ for all $t \geq T_{1}$, then $I(t)$ in system (1.1) is permanent.

Before we give the proof of Theorem 4.1, we first prove the following lemma.

Lemma 4.1. If there exist constants $\lambda>0, p>0$ and $T_{1}>0$ such that (4.1), (4.2) and $G(p, t)>0$ hold for all $t \geq T_{1}$. Then there exists $T_{2}>T_{1}$ so that $W(p, t) \leq 0$ for all $t \geq T_{2}$.

Proof: From Lemma 2.2, we consider the following two cases:

(i) $W(p, t)>0$ for all $t \geq T_{2}$;

(ii) $W(p, t) \leq 0$ for all $t \geq T_{2}$.

Suppose $W(p, t)>0$ for all $t \geq T_{2}$, then we have $E(t)>\frac{I(t)}{p}$ for all $t \geq T_{2}$. From the third equation of system (1.1), we have

$$
\begin{aligned}
\frac{\mathrm{d} I(t)}{\mathrm{d} t} & >\varepsilon(t) \frac{1}{p} I(t)-(\mu(t)+\delta(t)+d(t)) I(t) \\
& =I(t)\left\{\varepsilon(t) \frac{1}{p}-(\mu(t)+\delta(t)+d(t))\right\} .
\end{aligned}
$$

So we obtain

$$
I(t)>I\left(T_{2}\right) \exp \left(\int_{T_{2}}^{t}\left\{\frac{\varepsilon(s)}{p}-\mu(s)-\delta(s)-d(s)\right\} \mathrm{d} s\right)
$$

for all $t \geq T_{2}$. From the inequality (4.2), there exist positive constants $\eta>0$ and $T>0$ such that

$$
\int_{t+\lambda}^{t}\left\{\varepsilon(s) \frac{1}{p}-(\mu(s)+\delta(s)+d(s))\right\} \mathrm{d} s>\eta
$$

for all $t \geq T$. So the inequality (4.3) holds for all $t \geq \max \left\{T_{2}, T\right\}$. Then $\lim _{t \rightarrow+\infty} I(t)=+\infty$, which contradicts with the boundedness of $I(t)$ in Lemma 2.1. Now, we prove Theorem 4.1 by using Lemma 4.1 .

Proof: For simplicity, let $m_{\epsilon}:=m-\epsilon, M_{\epsilon}:=M+\epsilon$, where $\epsilon>0$ is a constant. In fact, Lemma 2.1 implies that for any sufficiently small $\epsilon>0$, there exists $T>0$ such that

$$
m_{\epsilon}<N_{*}(t) \leq N^{*}(t)<M_{\epsilon}
$$

for all $t \geq T$. The inequality (4.1) implies that for any sufficiently small $\eta>0$, there exists $T_{1}>T$ such that

$$
\int_{t}^{t+\lambda}\left\{\frac{p \beta(s) N_{*}(s)}{1+\alpha N_{*}(s)}-(\mu(s)+\varepsilon(s)+\gamma(s))\right\} \mathrm{d} s>\eta
$$


for all $t \geq T_{1}$. We define

$$
\begin{aligned}
& \beta^{+}:=\sup _{t \geq 0} \beta(t), \mu^{+}:=\sup _{t \geq 0} \mu(t), \delta^{+}:=\sup _{t \geq 0} \delta(t), \\
& d^{+}:=\sup _{t \geq 0} d(t), \varepsilon^{+}:=\sup _{t \geq 0} \varepsilon(t), \gamma^{+}:=\sup _{t \geq 0} \gamma(t) .
\end{aligned}
$$

Thus, by (4.5) and (4.6), for any sufficiently small $\eta_{1}<\eta$ and $T_{2}>T_{1}$, there exist very small $\epsilon_{i}, \quad i \in\{1,2,3\}$ such that

$$
\begin{gathered}
\int_{t}^{t+\lambda}\left\{\frac{\beta(s)}{1+\alpha N_{*}(s)}\left(N_{*}(s)-\epsilon_{1}-k \epsilon_{2}\right) p-(\mu(s)+\varepsilon(s)+\gamma(s))\right\} \mathrm{d} s>\eta_{1}, \\
N_{*}(t)-\epsilon_{1}-k \epsilon_{2}>m_{\epsilon}
\end{gathered}
$$

for all $t \geq T_{2}$, where $k:=1+\frac{\beta^{+}}{1+\alpha M_{\epsilon}} M_{\epsilon} \omega_{2}$. Lemma 2.1 implies that for any sufficiently small $\epsilon_{2}>0$, we have

$$
\int_{t_{1}}^{t_{1}+\omega_{2}}\left\{\frac{\beta(s) M_{\epsilon} \epsilon_{2}}{1+\alpha M_{\epsilon}}-(\mu(s)+\varepsilon(s)+\gamma(s)) \epsilon_{1}\right\} \mathrm{d} s<-\eta_{1}
$$

for all $t \geq T_{2}$. First, we prove

$$
\limsup _{t \rightarrow+\infty} I(t)>\epsilon_{2}
$$

In fact, if it is not true, there exists $T_{3} \geq T_{2}$ such that

$$
I(t) \leq \epsilon_{2}
$$

for all $t \geq T_{3}$. If $E(t) \geq \epsilon_{1}$ for all $t \geq T_{3}$, then from (4.5) and (4.6), we have

$$
\begin{aligned}
& E(t) \\
& =E\left(T_{3}\right)+\int_{T_{3}}^{t}\left\{\frac{\beta(s) I(s)}{1+\alpha S(s)}(N(s)-E(s)-I(s))-(\mu(s)+\varepsilon(s)+\gamma(s)) E(s)\right\} \mathrm{d} s \\
& \leq E\left(T_{3}\right)+\int_{T_{3}}^{t}\left\{\frac{\beta(s)}{1+\alpha M_{\epsilon}} M_{\epsilon} \epsilon_{2}-(\mu(s)+\varepsilon(s)+\gamma(s)) \epsilon_{1}\right\} \mathrm{d} s
\end{aligned}
$$

for all $t \geq T_{3}$. It follows from inequality (4.9) that $\lim _{t \rightarrow+\infty} E(t)=-\infty$. This contradicts with the boundedness of solution. Hence, there exists an $s_{1} \geq T_{3}$ such that $E\left(s_{1}\right)<\epsilon_{1}$. In the following we prove

$$
E(t) \leq \epsilon_{1}+\frac{\beta^{+}}{1+\alpha M_{\epsilon}} M_{\epsilon} \omega_{2} \epsilon_{2}
$$

for all $t \geq s_{1}$. If it is not true, there exists an $s_{2}>s_{1}$ such that

$$
E\left(s_{2}\right)>\epsilon_{1}+\frac{\beta^{+}}{1+\alpha M_{\epsilon}} M_{\epsilon} \omega_{2} \epsilon_{2} .
$$

Hence, there necessarily exists an $s_{3} \in\left(s_{1}, s_{2}\right)$ such that $E\left(s_{3}\right)=\epsilon_{1}$ and $E(t)>\epsilon_{1}$ for all $t \in\left(s_{3}, s_{2}\right)$. Let $n \geq 0$ be an integer such that $s_{2} \in\left[s_{3}+n \omega_{2}, s_{3}+(n+1) \omega_{2}\right]$. By (4.9), we obtain 


$$
\begin{aligned}
& E\left(s_{2}\right) \\
& =E\left(s_{3}\right)+\int_{s_{2}}^{s_{3}}\left\{\frac{\beta(s) I(s)}{1+\alpha S(s)}\left(N^{*}(s)-E(s)-I(s)\right)-(\mu(s)+\varepsilon(s)+\gamma(s)) E(s)\right\} \mathrm{d} s \\
& <\epsilon_{1}+\left\{\int_{s_{3}}^{s_{3}+n \omega_{2}}+\int_{s_{3}+n \omega_{2}}^{s_{2}}\right\}\left\{\frac{\beta(s) M_{\epsilon} \epsilon_{2}}{1+\alpha M_{\epsilon}}-(\mu(s)+\varepsilon(s)+\gamma(s)) \epsilon_{1}\right\} \mathrm{d} s \\
& <\epsilon_{1}+\int_{s_{3}+n \omega_{2}}^{s_{2}} \frac{\beta(s)}{1+\alpha M_{\epsilon}} M_{\epsilon} \epsilon_{2} \mathrm{~d} s \\
& <\epsilon_{1}+\frac{\beta^{+}}{1+\alpha M_{\epsilon}} M_{\epsilon} \omega_{2} \epsilon_{2},
\end{aligned}
$$

This contradicts with $E\left(s_{2}\right)>\epsilon_{1}+\frac{\beta^{+}}{1+\alpha M_{\epsilon}} M_{\epsilon} \omega_{2} \epsilon_{2}$. Hence, (4.11) is valid. By Lemma 4.1, there exists $T_{4} \geq s_{1}$ such that $W(p, t)=p E(t)-I(t) \leq 0$ for all $t \geq T_{4}$. Therefore, by (4.10) and (4.11), we have $E(t)+I(t) \leq \epsilon_{1}+k \epsilon_{2}$ for all $t \geq T_{4}$, then

$$
\begin{aligned}
\frac{\mathrm{d} E(t)}{\mathrm{d} t} & =\frac{\beta(t) I(t)(N(t)-E(t)-I(t))}{1+\alpha(N(t)-E(t)-I(t))}-(\mu(t)+\varepsilon(t)+\gamma(t)) E(t) \\
& \geq \frac{\beta(t) I(t)\left(N_{*}(t)-E(t)-I(t)\right)}{1+\alpha\left(N_{*}(t)-E(t)-I(t)\right)}-(\mu(t)+\varepsilon(t)+\gamma(t)) E(t) \\
& \geq \frac{\beta(t) E(t)\left(N_{*}(t)-E(t)-I(t)\right) p}{1+\alpha N_{*}(t)}-(\mu(t)+\varepsilon(t)+\gamma(t)) E(t) \\
& \geq E(t)\left\{\frac{\beta(t)\left(N_{*}(t)-E(t)-I(t)\right) p}{1+\alpha N_{*}(t)}-(\mu(t)+\varepsilon(t)+\gamma(t))\right\} \\
& \geq E(t)\left\{\frac{\beta(t)\left(N_{*}(t)-\epsilon_{1}-k \epsilon_{2}\right) p}{1+\alpha N_{*}(t)}-(\mu(t)+\varepsilon(t)+\gamma(t))\right\} .
\end{aligned}
$$

We obtain

$$
E(t) \geq E\left(T_{4}\right) \exp \left(\int_{T_{4}}^{t}\left\{\frac{\beta(s)\left(N_{*}(s)-\epsilon_{1}-k \epsilon_{2}\right) p}{1+\alpha N_{*}(s)}-(\mu(s)+\varepsilon(s)+\gamma(s))\right\} \mathrm{d} s\right) .
$$

By (4.7) we obtain $\lim _{t \rightarrow+\infty} E(t)=+\infty$. This contradicts with Lemma 2.1 ( $E(t)$ is uniformly bounded). Hence, $\limsup _{t \rightarrow+\infty} I(t)>\epsilon_{2}$ is true.

Next, we prove

$$
\liminf _{t \rightarrow+\infty} I(t) \geq I_{1},
$$

where $I_{1}>0$ is a constant given in the following lines. By inequality (4.7), (4.8), (4.9) and Lemma 2.1, there exist $\tilde{T}_{3}\left(\geq T_{2}\right), \lambda_{2}>0, \eta_{2}>0$ such that $\lambda_{3} \geq \lambda_{2}$ and $t \geq \tilde{T}_{3}$, we obtain

$$
\begin{gathered}
\int_{t}^{t+\lambda_{3}}\left\{\frac{\beta(s)}{1+\alpha N_{*}(s)}\left(N_{*}(s)-\epsilon_{1}-k \epsilon_{2}\right) p-(\mu(s)+\varepsilon(s)+\gamma(s))\right\} \mathrm{d} s>\eta_{2}, \\
\int_{t_{1}}^{t_{1}+\lambda_{3}}\left\{\frac{\beta(s) M_{\epsilon} \epsilon_{2}}{1+\alpha M_{\epsilon}}-(\mu(s)+\varepsilon(s)+\gamma(s)) \epsilon_{1}\right\} \mathrm{d} s<-M_{\epsilon},
\end{gathered}
$$




$$
\int_{t_{1}}^{t_{1}+\lambda_{3}} \beta(s) \mathrm{d} s>\eta_{2}
$$

Let $C>0$ be a constant satisfying

$$
\mathrm{e}^{-\left(\mu^{+}+\varepsilon^{+}+\gamma^{+}\right) \lambda_{2}} \frac{m_{\epsilon} v_{2} \eta_{2} \mathrm{e}^{n^{*} \eta_{2}}}{1+\alpha M_{\epsilon}}>\epsilon_{1}+\frac{\beta^{+} M_{\epsilon} \omega_{2} \epsilon_{2}}{1+\alpha M_{\epsilon}}
$$

where $v_{2}=\epsilon_{2} \mathrm{e}^{-\left(\mu^{+}+\delta^{+}+d^{+}\right) 2 \lambda_{2}}, n^{*} \leq \frac{C}{\lambda_{2}}$.

Because we have proved $\lim \sup _{t \rightarrow+\infty} I(t)>\epsilon_{2}$, there are only two possibilities as follows:

(i) There exists $\tilde{T}_{4} \geq \tilde{T}_{3}$, then as $t \geq \tilde{T}_{4}$, we obtain $I(t) \geq \epsilon_{2}$;

(ii) $I(t)$ oscillates about $\epsilon_{2}$ for all large $t$.

In case (i), we have $\liminf _{t \rightarrow+\infty} I(t) \geq \epsilon_{2}=: I_{1}$. In case (ii), there necessarily exist $t_{1}, t_{2} \geq \tilde{T}_{3}\left(t_{2} \geq t_{1}\right)$ such that

$$
\left\{\begin{array}{l}
I\left(t_{1}\right)=I\left(t_{2}\right)=\epsilon_{2}, \\
I(t)<\epsilon_{2}, \quad t \in\left(t_{1}, t_{2}\right) .
\end{array}\right.
$$

Suppose that $t_{2}-t_{1} \leq C+2 \lambda_{2}$. Then

$$
\frac{\mathrm{d} I(t)}{\mathrm{d} t} \geq-\left(\mu^{+}+\delta^{+}+d^{+}\right) I(t)
$$

which implies

$$
\begin{aligned}
I(t) & \geq I\left(t_{1}\right) \exp \left(\int_{t_{1}}^{t}-\left(\mu^{+}+\delta^{+}+d^{+}\right) \mathrm{d} s\right) \\
& \geq \epsilon_{2} \mathrm{e}^{-\left(\mu^{+}+\delta^{+}+d^{+}\right)\left(C+2 \lambda_{2}\right)}:=I_{1} .
\end{aligned}
$$

for all $t \in\left(t_{1}, t_{2}\right)$. Suppose that $t_{2}-t_{1}>C+2 \lambda_{2}$. Then

$$
I(t) \geq \epsilon_{2} \mathrm{e}^{-\left(\mu^{+}+\delta^{+}+d^{+}\right)\left(C+2 \lambda_{2}\right)}=I_{1}
$$

for all $t \in\left(t_{1}, t_{1}+C+2 \lambda_{2}\right)$. Now we only prove $I(t) \geq I_{1}$ for all $t \in\left[t_{1}+C+2 \lambda_{2}, t_{2}\right)$. If $E(t) \geq \epsilon_{1}$ for all $t \in\left[t_{1}, t_{1}+\lambda_{2}\right]$. By the second equation of system (1.1) and inequality (4.13), we have

$$
\begin{aligned}
E\left(t_{1}+\lambda_{2}\right) & \leq E\left(t_{1}\right)+\int_{t_{1}}^{t_{1}+\lambda_{2}}\left\{\frac{\beta(s) M_{\epsilon}}{1+\alpha M_{\epsilon}} \epsilon_{2}+(\mu(s)+\varepsilon(s)+\gamma(s)) \epsilon_{1}\right\} \mathrm{d} s \\
& <M_{\epsilon}-M_{\epsilon}=0,
\end{aligned}
$$

which is contradiction. Hence, there exists an $s_{4} \in\left[t_{1}, t_{1}+\lambda_{2}\right]$ such that $E\left(s_{4}\right)<\epsilon_{1}$. We obtain that for $t \geq s_{4}$,

$$
E(t) \leq \epsilon_{1}+\frac{\beta^{+}}{1+\alpha M_{\epsilon}} M_{\epsilon} \omega_{2} \epsilon_{2} .
$$

By inequality (4.16), then for $t \in\left[t_{1}, t_{1}+2 \lambda_{2}\right]$

$$
I(t) \geq v_{2}=\epsilon_{2} \mathrm{e}^{-\left(\mu^{+}+\delta^{+}+d^{+}\right) 2 \lambda_{2}} .
$$

Therefore, by the second equation of system (1.1) and inequalities (4.8), (4.17), (4.18), we obtain that 


$$
\begin{aligned}
\frac{\mathrm{d} E(t)}{\mathrm{d} t} & =\frac{\beta(t) I(t)(N(t)-E(t)-I(t))}{1+\alpha(N(t)-E(t)-I(t))}-(\mu(t)+\varepsilon(t)+\gamma(t)) E(t) \\
& \geq \frac{\beta(t) I(t)}{1+\alpha M_{\epsilon}}\left(N_{*}(t)-E(t)-I(t)\right)-(\mu(t)+\varepsilon(t)+\gamma(t)) E(t) \\
& \geq \frac{\beta(t)}{1+\alpha M_{\epsilon}} m_{\epsilon}-\left(\mu^{+}+\varepsilon^{+}+\gamma^{+}\right) E(t) .
\end{aligned}
$$

for all $t \in\left[t_{1}+\lambda_{2}, t_{1}+2 \lambda_{2}\right]$. By (4.14), we have

$$
\begin{aligned}
E(t) \geq & \mathrm{e}^{-\left(\mu^{+}+\varepsilon^{+}+\gamma^{+}\right)\left(t_{1}+2 \lambda_{2}\right)}\left\{E\left(t_{1}+\lambda_{2}\right) \mathrm{e}^{\left(\mu^{+}+\varepsilon^{+}+\gamma^{+}\right)\left(t_{1}+\lambda_{2}\right)}\right. \\
& \left.+\int_{t_{1}+\lambda_{2}}^{t_{1}+2 \lambda_{2}} \frac{\beta(s)}{1+\alpha M_{\epsilon}} m_{\epsilon} \nu_{2} \mathrm{e}^{\left(\mu^{+}+\varepsilon^{+}+\gamma^{+}\right) s} \mathrm{~d} s\right\} \\
\geq & \mathrm{e}^{-\left(\mu^{+}+\varepsilon^{+}+\gamma^{+}\right)\left(t_{1}+2 \lambda_{2}\right)} \int_{t_{1}+\lambda_{2}}^{t_{1}+2 \lambda_{2}} \frac{\beta(s)}{1+\alpha M_{\epsilon}} m_{\epsilon} \nu_{2} \mathrm{e}^{\left(\mu^{+}+\varepsilon^{+}+\gamma^{+}\right) s} \mathrm{~d} s \\
\geq & \mathrm{e}^{-\left(\mu^{+}+\varepsilon^{+}+\gamma^{+}\right) \lambda_{2}} \frac{1}{1+\alpha M_{\epsilon}} m_{\epsilon} \nu_{2} \eta_{2} .
\end{aligned}
$$

Now, we suppose there exists $t_{0}>0$ such that $t_{0} \in\left(t_{1}+C+2 \lambda_{2}, t_{2}\right)$, then $I\left(t_{0}\right)=I_{1}$ and $I(t) \geq I_{1}$ for all $t \in\left[t_{1}, t_{0}\right]$. By Lemma 4.1, we assume that $t_{1}$ is so large that $W(p, t)=p E(t)-I(t) \leq 0$ for all $t \geq t_{1}+2 \lambda_{2}$. Hence, by (4.8), we further have

$$
\begin{aligned}
\frac{\mathrm{d} E(t)}{\mathrm{d} t} & =\frac{\beta(t) I(t)(N(t)-E(t)-I(t))}{1+\alpha(N(t)-E(t)-I(t))}-(\mu(t)+\varepsilon(t)+\gamma(t)) E(t) \\
& \geq E(t)\left\{\frac{\beta(t)\left(N_{*}(t)-\epsilon_{1}-k \epsilon_{2}\right) p}{1+\alpha N_{*}(t)}-(\mu(t)+\varepsilon(t)+\gamma(t))\right\}
\end{aligned}
$$

for all $t \in\left(t_{1}+2 \lambda_{2}, t_{2}\right)$. By (4.12) and (4.19), we have

$$
\begin{aligned}
E(t) & E\left(t_{1}+2 \lambda_{2}\right) \exp \left(\int_{t_{1}+2 \lambda_{2}}^{t_{0}}\left\{\frac{\beta(t)\left(N_{*}(t)-\epsilon_{1}-k \epsilon_{2}\right) p}{1+\alpha N_{*}(t)}-(\mu(t)+\varepsilon(t)+\gamma(t))\right\} \mathrm{d} s\right) \\
= & E\left(t_{1}+2 \lambda_{2}\right) \exp \left(\left\{\int_{t_{1}+2 \lambda_{2}}^{t_{1}+2 \lambda_{2}+\lambda_{2}}+\int_{t_{1}+2 \lambda_{2}+\lambda_{2}}^{t_{1}+2 \lambda_{2}+2 \lambda_{2}}+\cdots\right.\right. \\
& \left.\left.+\int_{t_{1}+2 \lambda_{2}+\left(n^{*}-1\right) \lambda_{2}}^{t_{0}}\right\}\left\{\frac{\beta(t)\left(N_{*}(t)-\epsilon_{1}-k \epsilon_{2}\right) p}{1+\alpha N_{*}(t)}-(\mu(t)+\varepsilon(t)+\gamma(t))\right\} \mathrm{d} s\right) \\
\geq & \mathrm{e}^{-\left(\mu^{+}+\varepsilon^{+}+\gamma^{+}\right) \lambda_{2}} \frac{1}{1+\alpha M_{\epsilon}} m_{\epsilon} v_{2} \eta_{2} \mathrm{e}^{n^{*} \eta_{2}} .
\end{aligned}
$$

Thus, by (4.17), we have

$$
\varepsilon_{1}+\frac{\beta^{+} M_{\epsilon} \omega_{2} \epsilon_{2}}{1+\alpha M_{\epsilon}} \geq \mathrm{e}^{-\left(\mu^{+}+\varepsilon^{+}+\gamma^{+}\right) \lambda_{2}} \frac{m_{\epsilon} \nu_{2} \eta_{2} \mathrm{e}^{n^{*} \eta_{2}}}{1+\alpha M_{\epsilon}},
$$

This contradicts with (4.15). Hence, $I(t) \geq I_{1}$ for all $t \in\left[t_{1}+C+2 \lambda_{2}, t_{2}\right]$, which implies $\liminf _{t \rightarrow+\infty} I(t) \geq I_{1}$. Thus, the infectious population of system 
(1.1) is permanent.

\section{Numerical Simulations}

Numerical verification of the results is necessary for completeness of the analytical study. In Sections 3 and 4, we focused our attention on the dynamic analysis of system (1.1). In the present section, numerical simulations are carried out to illustrate the analytical results of system (1.1) by means of the software Matlab.

In order to testify the validity of our results, in system (1.1), fix $\Lambda=1$, $\beta(t)=0.3+0.1 \cos t, \quad \alpha=0.3, \quad \mu=0.1, \delta=0.1, \quad \gamma=0.8, \quad \epsilon=0.2, d=0.5$. Then, from system (1.1), we have $\lim _{t \rightarrow+\infty} N^{*}(t)=1$. We easily verify that assumptions (H1) and (H2) hold.We choose $\lambda=1$ and $p=2$. Then we have

$$
\begin{aligned}
& R_{1}(\lambda, p):=\int_{0}^{1}\left\{2 \frac{0.3+0.1 \cos t}{1+0.3}-(0.1+0.2+0.8)\right\} \mathrm{d} t \approx-0.84<0, \\
& R_{1}^{*}(\lambda, p):=\int_{0}^{1}\left\{0.2 \frac{1}{2}-(0.1+0.1+0.5)\right\} \mathrm{d} t=-0.7<0 .
\end{aligned}
$$

and

$$
\begin{aligned}
G(p, t) & :=\int_{0}^{1}\left\{2 \frac{0.3+0.1 \cos t}{1+0.3}+0.1+0.5-0.8-\left(1+\frac{1}{2}\right) 0.2\right\} \mathrm{d} t \\
& \approx-0.1385<0 .
\end{aligned}
$$

for all $t>0$. From Theorem 3.1, we see that the infectious population of system (1.1) is extinct, see Figure 1.

Fix $\Lambda=1, \quad \beta(t)=0.6+0.1 \cos t, \quad \alpha=0.6, \quad \mu=0.1, \delta=0.02, \quad \gamma=0.1$, $\epsilon=0.5, d=0.02$. We choose $\lambda=1$ and $p=2$. Then we have

$$
\begin{aligned}
& R_{2}(\lambda, p):=\int_{0}^{1}\left\{2 \frac{0.6+0.1 \cos t}{1+0.6}-(0.1+0.5+0.1)\right\} \mathrm{d} t \approx 0.05>0, \\
& R_{2}^{*}(\lambda, p):=\int_{0}^{1}\left\{0.5 \frac{1}{2}-(0.1+0.02+0.02)\right\} \mathrm{d} t=0.11>0 .
\end{aligned}
$$

and

$$
\begin{aligned}
G(p, t) & :=\int_{0}^{1}\left\{2 \frac{0.6+0.1 \cos t}{1+0.6}+0.02+0.02-0.1-\left(1+\frac{1}{2}\right) 0.5\right\} \mathrm{d} t \\
& \approx-0.06<0 .
\end{aligned}
$$

for all $t>T_{1}$. From Theorem 4.1, we see that the infectious population of system (1.1) is permanent, see Figure 2.

\section{Conclusions}

In this paper we obtain new sufficient conditions for the permanence and extinction of system (1.1). We prove that our conditions give the threshold-type result by the basic reproduction number given as in (3.1) when every parameter is given as a constant parameter. Thus our result is an extension result of the threshold-type result in the autonomous system. Our results may contribute to predicting the disease dynamics, such as permanence and extinction of the infectious population, when the phenomena are modeled as a nonautonomous system. 

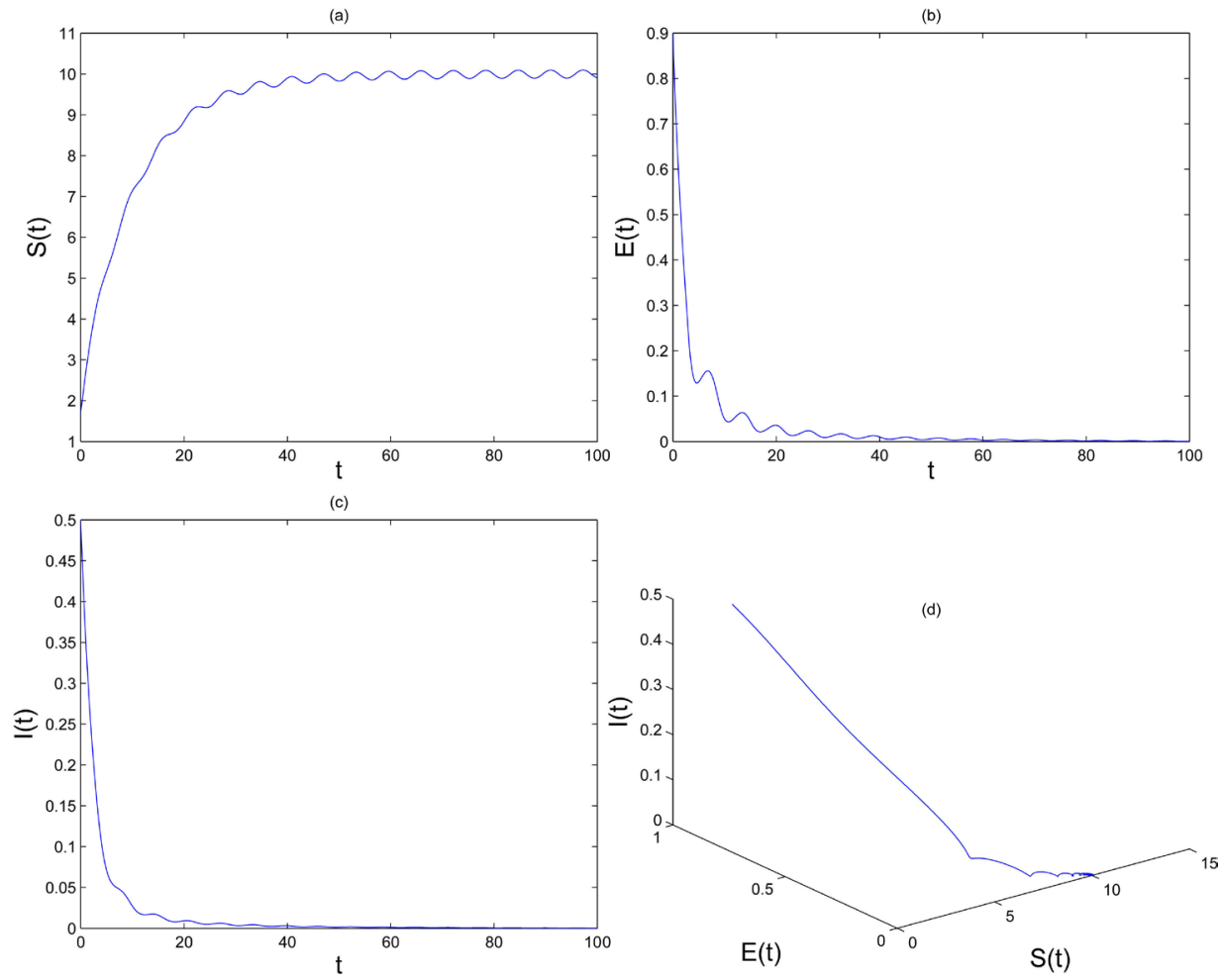

Figure 1. The trajectories of deterministic system (1.1) with $R_{1}(\lambda, p)<0, R_{1}^{*}(\lambda, p)<0, G(p, t)<0$. (a) Time series diagram of susceptible population, (b) Time series diagram of exposed population, (c) Time series diagram of infectious population, (d) phase diagram of three populations (susceptible, exposed, infectious), respectively.
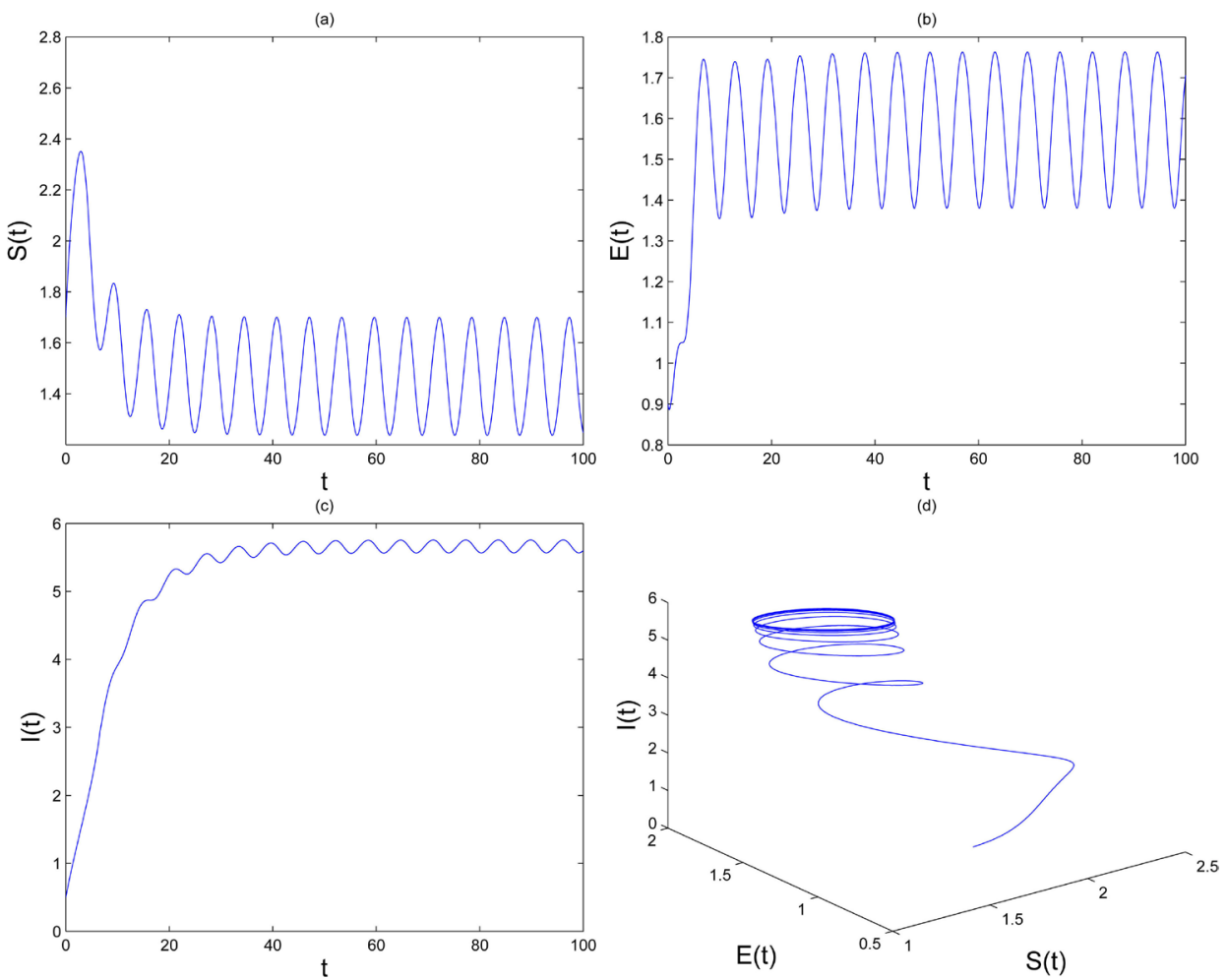

Figure 2. The trajectories of deterministic system (1.1) with $R_{1}(\lambda, p)>0, R_{1}^{*}(\lambda, p)>0, G(p, t)<0$. The meaning of (a) (d) is similar to Figure 1. 
In Section 5, we provide numerical examples to illustrate the validity of our results. In those examples we show that conditions in Theorems 4.1 for the permanence and extinction of infectious population of system (1.1) are not satisfied. One may argue that our conditions for the permanence and extinction may not sharp.

It is still an open problem that if the basic reproduction number for (1.1) works as a threshold parameter to determine the permanence and extinction of infectious population like in the autonomous system.

\section{Acknowledgements}

The research has been partially supported by the Natural Science Foundation of China (No. 11561004).

\section{Conflicts of Interest}

The authors declare no conflicts of interest regarding the publication of this paper.

\section{References}

[1] (2011) Schistosomiasis (Bilharzia). NHS Choices.

[2] Fenwick, A. (2012) The Global Burden of Neglected Tropical Diseases. Public Health, 126, 233-236. https://doi.org/10.1016/j.puhe.2011.11.015

[3] Lozano, R., Naghavi, M., Foreman, K., Lim, S., Shibuya, K., Aboyans, V., Abraham, J., Adair, T., et al. (2012) Global and Regional Mortality from 235 Causes of Death for 20 Age Groups in 1990 and 2010: A Systematic Analysis for the Global Burden of Disease Study 2010. The Lancet, 380, 2095-2128. https://doi.org/10.1016/S0140-6736(12)61728-0

[4] Thtiot-Laurent, S.A., Boissier, J., Robert, A. and Meunier, B. (2013) Schistosomiasis Chemotherapy. Angewandte Chemie, 52, 7936-7956. https://doi.org/10.1002/anie.201208390

[5] World Health Organization (2014) Schistosomiasis Fact Sheet N115.

[6] World Health Organization Schistosomiasis a Major Public Health Problem.

[7] The Carter Center Schistosomiasis Control Program.

[8] Gao, S., Liu, Y., Luo, Y. (2011) Control Problems of a Mathematical Model for Schistosomiasis Transmission Dynamics. Nonlinear Dynamics, 63, 503-512. https://doi.org/10.1007/s11071-010-9818-Z

[9] Feng, Z., Li, C. and Milner, F.A. (2005) Schistosomiasis Models with Two Migrating Human Groups. Mathematical and Computer Modelling, 41, 1213-1230. https://doi.org/10.1016/j.mcm.2004.10.023

[10] Qi, L. and Cui, J. (2012) The Delayed Barbours Model for Schistosomiasis. International Journal of Biomathematics, 5, Article ID: 1250024. https://doi.org/10.1142/S1793524511001660

[11] Anderson, R.M. and May, R.M. (1979) Prevalence of Schistosome Infections within Molluscan Populations: Observed Patterns and Theoretical Predictions. Parasitology, 79, 63-94. https://doi.org/10.1017/S0031182000051982

[12] Zhang, P., Feng, Z. and Milner, F.A. (2007) A Schistosomiasis Model with an Age-Structure in Human Hosts and Its Application to Treatment Strategies. Ma- 
thematical Biosciences, 205, 83-107. https://doi.org/10.1016/j.mbs.2006.06.006

[13] Castillo-Chavez, C. and Thieme, H.R. (1995) Asymptotically Autonomous Epidemic Models. In: Arino, O. and Kimmel, M., Eds., Proceedings of the 3 rd International Conference on Mathematical Population Dynamics, 33.

[14] Thieme, H.R. (2009) Spectral Bound and Reproduction Number for Infinite-Dimensional Population Structure and Time Heterogeneity. SIAM Journal on Applied Mathematics, 70, 188-211. https://doi.org/10.1137/080732870

[15] Wang, X., Tao, Y. and Song, X. (2009) Analysis of Pulse Vaccination Strategy in SIRVS Epidemic Model. Communications in Nonlinear Science and Numerical Simulation, 14, 2747-2456. https://doi.org/10.1016/j.cnsns.2008.10.022

[16] Wang, W. and Zhao, X. (2008) Threshold Dynamics for Compartmental Epidemic Models in Periodic Environments. Journal of Dynamics and Differential Equations, 20, 699-717. https://doi.org/10.1007/s10884-008-9111-8

[17] Zhang, T., Liu, J. and Teng, Z. (2008) Differential Susceptibility Time-Dependent SIR Epidemic Model. International Journal of Biomathematics, 1, 45-64. https://doi.org/10.1142/S1793524508000059

[18] Zhang, T., Teng, Z. and Gao, S. (2008) Threshold Conditions for a Non-Autonomous Epidemic Model with Vaccination. Applicable Analysis, 87, 181-199. https://doi.org/10.1080/00036810701772196

[19] Zhang, F. and Zhao, X. (2007) A Periodic Epidemic Model in a Patchy Environment. Journal of Mathematical Analysis and Applications, 325, 496-516. https://doi.org/10.1016/j.jmaa.2006.01.085

[20] Zhang, T. and Teng, Z. (2007) On a Nonautonomous SEIRS Model in Epidemiology. Bulletin of Mathematical Biology, 69, 2537-2559. https://doi.org/10.1007/s11538-007-9231-Z

[21] Wang, X.H., Wu, X.H. and Zhou, X.N. (2006) Bayesian Estimation of Community Prevalences of Schistosoma japonicum Infection in China. International Journal for Parasitology, 36, 895-902. https://doi.org/10.1016/j.ijpara.2006.04.003

[22] Vandemark, L.M., Jia, T.W. and Zhou, X.N. (2010) Social Science Implications for Control of Helminth Infections in Southeast Asia. Advances in Parasitology, 73, 137-170. https://doi.org/10.1016/S0065-308X(10)73006-2 